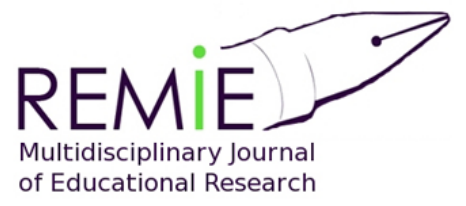

Instructions for authors, subscriptions and further details:

http://remie.hipatiapress.com

\title{
Decálogo De Competencias y Sus Indicadores Para Gestión De Capital Humano Universitario
}

\author{
Azucena Leticia Herrera Aguado ${ }^{1}$ \\ 1) Universidad Tecnológica de Puebla. México
}

Date of publication: February $15^{\text {th }}, 2018$

Edition period: February 2018-May 2018

To cite this article: Herrera, A.L. (2018). Decálogo de Competencias y sus Indicadores para Gestión de Capital Humano Universitario. Multidisciplinary Journal of Educational Research, 8(1), 56-85. doi:

10.17583/remie.2018.2990

To link this article: http://dx.doi.org/10.17583/remie.2018.2990

\section{PLEASE SCROLL DOWN FOR ARTICLE}

The terms and conditions of use are related to the Open Journal System and to Creative Commons Attribution License (CC-BY). 


\title{
Decálogo de Competencias y sus Indicadores para Gestión de Capital Humano Universitario
}

\author{
Azucena Leticia Herrera \\ Universidad Tecnológica de \\ Puebla
}

\section{Abstract}

La presente investigación es cuantitativa y se enfoca a analizar las percepciones de una muestra de sujetos participantes en programas de posgrado de la Benemérita Universidad Autónoma de Puebla, en torno a los indicadores de competencias que deben estar presentes en el perfil del docente.

Los hallazgos del presente consisten en un modelo de perfil de puesto con los indicadores de competencias que debe poseer el docente de posgrado, agrupados en un decálogo de competencias.

El modelo expuesto es un referente innovador de la teoría de competencias docentes para diseño de políticas y programas aplicables a personal docente universitario, bajo condiciones de eficacia, uniformidad y objetividad; su utilidad radica en que apuntala las funciones de Administración de Capital Humano (ACH) en Instituciones de Educación Superior (IES), especialmente las correspondientes a la gestión del perfil docente que fomentan la calidad del proceso educativo en programas de posgrado de cualquier institución que oferte ese nivel, en concordancia con estándares internacionales.

Keywords: competencias, educación, gestión capital humano, diseño de puesto. 


\title{
Decalogue of Competences and its Indicators for the Management of Academic Staff in Universities
}

\author{
Azucena Leticia Herrera \\ Universidad Tecnológica de
}

Puebla

\section{Resumen}

The present research is quantitative and focuses on the analysis of the perceptions of a sample of participants in postgraduate programs of the Autonomous University of Puebla, around the indicators of competencies that must be present in the profile of the university professor.

The results consist of a profile model with the indicators of competences that must have the university professor, grouped in a decalogue of competences.

The model presented is an innovative reference of the theory of competence applied to the design of policies and programs for university teachers, in conditions of efficiency, uniformity and objectivity; Its usefulness is that it underlies the functions of the Human Capital Administration (ACH) in the Universities (IES), especially those functions corresponding to the management of the teaching profile that promote the quality of the educational process in the postgraduate programs of any institution in accordance with international standards.

Palabras clave: competences, education, personnel management, job design 


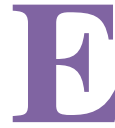

n el contexto de la Administración de Capital Humano (ACH) la presente investigación pretende continuar la búsqueda de conocimiento relativa a modelos para gestión de personal basados en un perfil por competencias, que sean aplicables al docente de posgrado, obtenidos del análisis de la función de evaluación al desempeño del puesto docente que imparte en posgrado, en la Benemérita Universidad Autónoma de Puebla (BUAP).

El estudio que se desarrolla en el presente trabajo es cualitativo-cuantitativo y culmina con la exposición de resultados consistentes en la determinación de una generalización de indicadores de competencias para el perfil del docente de posgrado, asociados al decálogo de competencias en el perfil de ese actor, los cuales constituyen parámetros objetivos, uniformes y medibles, en concordancia con estándares internacionales y sustentados en bases científicas, para aplicarse en IES mexicanas y extranjeras, públicas y privadas.

\section{Marco Conceptual}

\section{Gestión de Personal Docente en las IES}

En materia de gestión de personal, la ventaja competitiva de una organización está positivamente relacionada con las capacidades de los recursos humanos (Lillo, et al., 2007), debido a la importancia que representan éstos al pleno desarrollo de su actividad (Uriel, et. al., 2001). En el mismo tenor la implementación de modelos de gestión por competencias en las organizaciones es un factor que garantiza el éxito y la supervivencia (Medina, et al., 2012), siendo el área de recursos humanos la más beneficiada, fundamentalmente en sus políticas de selección de personal, formación y desarrollo, evaluación del desempeño y estimulación. En ese sentido, las competencias laborales son responsabilidad de los procesos de gestión de recursos humanos (Lindgren, et al. 2004), y es McClelland (1973) quien atribuye el éxito de los individuos en su trabajo a las competencias que aparecen vinculadas a un perfil que causa un rendimiento superior. Con Cuesta (2005) se incluye a la gestión de competencias en el marco de actuación de la administración de los recursos humanos. 
De allí surge la necesidad de proporcionar a los administradores de personal en toda organización, incluyendo en este universo a las Instituciones de Educación Superior (IES), las guías que les permitan realizar de manera óptima la gestión del personal, aplicable a los cinco subsistemas cruciales en la competitividad organizacional, que a decir de Arias Galicia et. al. (1999) son: a) reclutar a las personas y seleccionarlas; b) integrarlas y orientarlas; c) hacerlas trabajar y desarrollarlas; d) recompensarlas y evaluarlas; y, e) controlarlas (entendida esta última como revisión o auditoría). Ninguna de estas cinco funciones puede realizarse adecuadamente si no se cuenta con un perfil de puesto adecuado y definido de antemano.

\section{Las Competencias y el Perfil del Personal Docente}

Según Tejedor y Jornet (2008), determinados comportamientos docentes pueden asociarse claramente con la consecución de objetivos deseables, y esos comportamientos deseables de una profesión se vinculan al término competencia profesional (Instituto Nacional de Empleo en España -INEM-, 1995; Bunk, 1994; Gonczy, 2001; Tejada, 2005; Zabala y Arnau, 2008), definida por la autora como la capacidad o habilidad de efectuar tareas o atender situaciones en contextos particulares, de forma eficaz, movilizando actitudes, aptitudes, habilidades y conocimientos de forma simultánea e interrelacionada para obtener resultados exitosos.

En el ámbito educativo, las teorías de competencias que son referente del presente son aplicables al docente en general (Comellas, 2002; Perrenoud, 2004; Zabalza, 2005; Cano, 2005; y Rueda, 2008 y 2009) y generalmente no especifican niveles educativos, salvo Bain (2007), quien enfoca su teoría al docente universitario, más no le otorga el enfoque de las competencias. Las mencionadas no se vinculan a las teorías de la ACH del docente (a través de su perfil de puesto) para atender urgencias como las señaladas por Marín (2002), cuando afirma que las competencias que posee el docente, podrá desarrollarlas en sus estudiantes.

Así, el enlace de la teoría de competencias y la teoría de gestión de personal es generada en posturas de investigaciones previas, entre las que se sostiene que la formación basada en competencias nos introduce de manera sistemática en la descripción de las actividades que se aplican en la 
resolución de problemas vinculados a un perfil profesional determinado, en los resultantes esperados y en los conocimientos que se vinculan en ellos Tejada (2005).

\section{La Competencia Genérica, la Competencia Específica y sus Plurales}

Es relevante que se refiera la postura de Zabalza (2005), quien sostiene que cada actividad suele exigir la presencia de un número variado de competencias, las cuales, a su vez, pueden ser desglosadas en unidades más específicas de competencia, en las que se especifican las tareas más concretas que están incluidas en la competencia global.

Con esos supuestos, en adelante, cuando se mencione la palabra competencia o su plural, se entenderá que cada competencia o grupo de ellas (plural) se integra, a su vez, de al menos una competencia (y regularmente más de una) que posee un carácter específico. Así, para los efectos del presente, cada competencia específica será denominada "indicador de competencia" y dos o más competencias específicas serán llamadas con su plural: indicadores de competencia. Cada indicador de competencia es independiente y puede contribuir a la consecución de más de una competencia global o genérica; cada indicador de competencia puede medirse a través de uno o más ítems.

\section{Requisitos de la Evaluación al Desempeño de Personal con Puesto Docente}

Según Tejedor y Jornet (2008), un sistema de evaluación al desempeño docente requiere determinar un modelo de profesor (comportamientos deseables y criterios básicos que deben orientar el proceso evaluativo) y las fuentes de información (profesor -auto informe y autoanálisis-, responsables académicos -decanos y directores- y estudiantes). Por su parte Echeverría (2002) (en Tejada, J., 2005), afirma que cualquier plan de evaluación de la competencia profesional exige la articulación de dispositivos válidos y fiables, con los cuales se pueda evidenciar que la competencia, inferida por el desempeño, se posee. 


\section{Evaluación de Personal Docente de Posgrado Basado en un Perfil por Competencias}

En la búsqueda de los indicadores de competencias idóneos que deben ser considerados para agruparse y luego precisar cada una de las diez competencias en el perfil del docente de posgrado, en el marco del modelo que es principal referente de esta investigación (Herrera, A., 2017 y 2010; y Herrera et. al., 2010a, 2010b y 2011), fueron imprescindibles las posturas de Zabalza (2005), Medina (2009), Morín (1999), Marín (2002). Igualmente, relevantes fueron las guías que en materia educativa han orientado acciones y estrategias del Espacio Europeo de Educación Superior (EEES, 1999 y 2009). Otros referentes cruciales han sido los diagnósticos y recomendaciones en materia de educación (competencias y perfil docente) emitidos por Banco de México (BM), Organización para la Cooperación y el Desarrollo (OCDE), Organización de las Naciones Unidas para la Educación, la Ciencia y la Cultura (UNESCO) y Organización de la Naciones Unidas (ONU) al gobierno mexicano (BUAP, 2006).

Cabe comentar que existen algunas limitantes en el proceso de evaluación al profesorado, puesto que pueden considerarse diversidad de finalidades y modelo de profesor ideal. Sin embargo, respecto a la evaluación del docente, para la autora, entendida como una función de ACH, hay coincidencias con Tejedor y Jornet (2008); Zabalza,_2005; Mobilia y García-Valcárcel, (1997) y López Mojarro (1999), ambos en Tejedor y Jornet (2008).

\section{La Evaluación de Personal Docente en la BUAP}

Con los antecedentes, se pretende que la presente investigación alcance la determinación de indicadores de competencia que integran cada una de las competencias del decálogo de Herrera (2017), a través del análisis de percepciones de una muestra, empleando la función de evaluación al desempeño del puesto docente de posgrado por el periodo 2010-2015, para obtener así un perfil de ese actor.

Partiendo de esos supuestos, y sin perder de vista que al menos 32 de las IES mexicanas son autónomas (una por cada estado -31- más una por la ciudad de México), se tomó como referente de este grupo a la Benemérita 
Universidad Autónoma de Puebla (BUAP) y a una muestra de sujetos participantes de sus posgrados para continuar con las investigaciones en materia de competencias del docente de posgrado.

La elección de esta IES ha sido en función de su representatividad a nivel nacional de sus homólogas, puesto que ya desde 2009, año anterior al que se inició este estudio, era una institución de educación pública y autónoma, poblano-mexicana, considerada dentro de los primeros seis lugares a nivel nacional con mayor número de posgrados (BUAP, 2009), contando a esa fecha 108 en todas las áreas del conocimiento. La distribución de estos programas de posgrado es de 29 pertenecientes al Programa Nacional de Posgrados de Calidad (PNPC) de CONACYT (2012) con 21 de maestría y ocho de doctorado, más 79 sin ese registro, de los cuales 50 de maestría, 14 de doctorado y 15 de especialidad.

Para cubrir los requerimientos de los mencionados programas, de la misma fuente se obtiene que la BUAP contaba a esa fecha con 2,129 Profesores de Tiempo Completo (PTC) y matrícula de 3480 alumnos inscritos en especialidades, maestrías y doctorados según el Anuario Estadístico (BUAP, 2010).

Como antecedentes de evaluación al desempeño docente en la BUAP se cuentan el Programa Institucional de Evaluación Académica (PIEVA) y el programa de Estímulos al Desempeño del Personal Académico (ESDEPED).

El Programa Institucional de Evaluación Académica (PIEVA, BUAP 2002) se ha ocupado de la evaluación institucional al desempeño docente en la BUAP desde 2002 en sus funciones áulicas, desde la percepción de los estudiantes y del propio docente (autoevaluación). En 2006 el instrumento fue actualizado por su Comisión con la finalidad de revitalizar el proceso de evaluación de la docencia y posteriormente en 2010, a petición de la Vicerrectoría de Investigación y Estudios de Posgrado (VIEP), la propia Comisión (consciente de las muchas limitaciones que planteaba la realidad institucional y derivado de los resultados incongruentes, obtenidos al aplicar el mismo instrumento a docentes que impartían en posgrado), se percató de la urgencia requerida en el diseño de un instrumento de evaluación que se ajustara a las particularidades de competencias de docentes que imparten en programas educativos de posgrado (BUAP, 2010). 
Por otra parte, también en materia de evaluación al desempeño de personal docente y su compensación con estímulos económicos (ambas funciones derivadas de gestión de personal docente), la BUAP (2008), al igual que la mayoría de las universidades públicas autónomas en México, se acoge al financiamiento federal que le otorga la Secretaría de Hacienda y Crédito Público -SHCP- (SHCP, 2002; SEP, 2013) para reconocer a sus docentes, previo concurso en convocatoria anual del Programa de Estímulos al Desempeño del Personal Docente (ESDEPED). Este programa contempla la evaluación de permanencia (antigüedad), 10\%; dedicación a la docencia (clase pizarrón), 30\%; calidad en el desempeño de las actividades académicas (investigación, gestión y extensión), 60\%. Derivado del otorgamiento de estímulo económico al desempeño docente por ESDEPED, en la BUAP se detectó una situación en el rubro de docencia: la institución no le proveía al docente que impartía en posgrado resultados de evaluación al desempeño, puesto que no había un instrumento institucional (PIEVA) que midiera esta función de $\mathrm{ACH}$ para quienes colaboraban en programas educativos de ese nivel, afectando a quienes contaban con la habilitación académica más alta (maestría y doctorado) en esa Universidad.

\section{Metodología}

La metodología seguida en este estudio, previa determinación de competencias que deben formar parte del perfil de puesto del docente que participa en programas educativos de posgrado, acorde a teorías expuestas, principalmente del modelo de Herrera (2017), se debe considerar en primera instancia el objetivo de la investigación, y, en segundo lugar, enlistar los principales pasos que guían su realización.

\section{Objetivo}

Establecer un modelo de competencias y sus indicadores de competencias asociados que apoye a la realización de funciones de administración del capital humano, sustentadas en una definición de perfil de puesto para el docente que participa en programas educativos de posgrado. 
Concretamente, la metodología seguida consiste de las siguientes acciones:

1. Búsqueda de parámetros estandarizados en la definición del perfil del docente universitario en México.

2. Análisis del perfil del docente requerido en posgrado en instituciones de educación superior.

3. Análisis, correlación y estructuración de un modelo de perfil docente de posgrado con base en competencias, que incluya indicadores claros y medibles, para aplicación en IES mexicanas y extranjeras. Este apartado constituye la parte central de este trabajo de investigación, ya que se enfoca a la estructuración de un modelo de perfil del docente de posgrado basado la teoría de las competencias, que permita llevar a cabo una gestión de recursos humanos apropiada al interior de cualquier IES.

4. Diseño de un instrumento que se aplique a una muestra de actores involucrados en programas educativos de posgrado de diversas áreas del conocimiento en la BUAP, con la finalidad de medir la validez del modelo propuesto.

5. Aplicación de instrumento para recabar las percepciones de los actores involucrados diversos programas de posgrado de la BUAP, pertenecientes a seis Dependencias de Educación Superior (DES), en el periodo 2010 a 2015.

6. Análisis de validez del modelo, empleando métodos estadísticos del programa SPSS como son análisis descriptivo, análisis de confiabilidad del instrumento y grupos de indicadores de competencias, análisis de confiabilidad de los ítems del instrumento (análisis de valoración), análisis de comparación y análisis factorial. Estos análisis estadísticos tienen por objetivo obtener información cuantitativa y determinar la validez del modelo de perfil de puesto docente propuesta, así como de cada indicador de competencia, para conocer la manera en que cada uno de ellos se agrupa a una competencia o más competencias, de aquellas previstas bajo el esquema de un decálogo de competencias; con la finalidad de ser empleadas para una adecuada $\mathrm{ACH}$ docente que imparte en posgrado. 


\section{Población y Muestra}

Acorde al Anuario Estadístico Institucional 2010 de la propia BUAP (2010a), el universo de participantes en posgrados es de 5,609 (100\%) y se integra por 3,480 alumnos matriculados en posgrado (62\%) y 2,129 PTC (38\%).

La distribución porcentual de los alumnos matriculados, por nivel de programa educativo en el posgrado era la siguiente: especialidad 32.04\%, maestría $59.83 \%$ y doctorado $8.13 \%$.

Se aclara que todos los PTC pueden participar en programas educativos de posgrado y, en el supuesto de que sólo participaran del posgrado los 698 docentes que al 2010 comprobaban pertenencia al Sistema Nacional de Investigadores (SNI), la participación porcentual por PTC en posgrado sería del $32.79 \%$.

Al aplicar la fórmula al universo para determinación de una muestra y tomando como desviación estándar el 0.5; con una confianza de la muestra del 95\% ( $Z=1.96)$; y, finalmente, considerando que pudiera presentarse un error muestral del 6.97\% (el límite superior aceptable es de 9\%), se obtiene que la muestra (n) debe ser de 191 individuos.

Habiendo determinado que una muestra representativa, adecuada y válida consistiría en 191 individuos participantes del posgrado de la BUAP, se definieron las variantes de aquellos sujetos que podrían participar en esta investigación, como a continuación se enlista:

a) Profesores de tiempo completo en activo o con puesto administrativo: directores de facultades, secretarios de investigación y estudios de posgrado, coordinadores de programas de posgrado, miembros del Consejo de Investigación y Estudios de Posgrado (CIEP) y Vicerrector de Investigación y Estudios de Posgrado (VIEP).

b) Estudiantes de posgrado. Inscritos en programas de especialidad, maestría o doctorado.

De lo anterior se deriva que el mínimo nivel de estudios que poseen los participantes es el de Licenciado.

Por otra parte, se debe recordar que las IES mexicanas cuentan con una agrupación convencional de programas educativos, en función del área del 
conocimiento a la que pertenecen bajo el nombre de Dependencias de Educación Superior (DES), entendida en estricto sentido con la acepción que se le otorga en el ámbito académico de las universidades autónomas mexicanas: el grupo de programas educativos, que bajo direcciones académicas o facultades, comparten un área del conocimiento. Por ello, se consideró como estrato de muestreo a cada una de las seis áreas del conocimiento asociadas a Dependencias de Educación Superior por áreas de conocimientos siguientes:

1. Ciencias Sociales y Económico-Administrativas.

2. Ciencias Exactas.

3. Ciencias Naturales.

4. Ingeniería y Tecnología.

5. Ciencias de la Salud.

6. Educación y Humanidades.

Lo anterior expuesto, obliga al investigador a realizar un muestreo aleatorio estratificado entre los individuos pertenecientes a cada una de las seis DES enlistadas, para asegurarse de que todos los estratos de interés estarán representados adecuadamente en la muestra.

Posteriormente se aplica el muestreo aleatorio simple al interior de cada DES, que ya no atiende a la distribución porcentual de matrícula de posgrado asentada en el segundo párrafo de este apartado de Población y muestra. Esta decisión de aplicación no estratificada no es imputable a quien realiza este estudio, sino que está en función de la autorización que la Vicerrectoría de Investigación y Estudios de Posgrado (VIEP) de la BUAP otorga para aplicación del muestreo a PTC y estudiantes pertenecientes a unidades académicas (Facultades) específicas.

Con esa restricción logística, el muestreo aleatorio simple, al interior de cada DES, permite que cualquier individuo que participe en posgrado de una de las seis DES en particular, sea considerado como idóneo para considerarse parte de la muestra.

Así, para obtener una muestra de 191 instrumentos válidos de la BUAP, se aplicaron 236 instrumentos a igual número de individuos (PTC y alumnos) participantes de posgrado de la BUAP. De los 236 instrumentos aplicados se tomaron como válidos 191 y se descartaron 45 puesto que no reunieron características de validez, la generalidad de ellos por no estar 
respondidos en forma completa o tener respuestas fuera de la escala que contempla el instrumento.

Puede afirmarse que los instrumentos aplicados cubrieron prácticamente la misma distribución porcentual de la población (matrícula de posgrado $62 \%$ y PTC $38 \%$ ), ya que, de los 191 instrumentos de la muestra, $61.78 \%$ se aplicaron entre alumnos de posgrado y $38.22 \%$ a PTC.

En cuanto a la estratificación aleatoria simple que deriva de autorizaciones de aplicación por la BUAP, se tiene que los 191 instrumentos requeridos por fórmula estadística se distribuyen por área del conocimiento (DES) como sigue: Ciencias Sociales y Económico-Administrativas (55), Ciencias Exactas (53), Ciencias Naturales (23), Ingeniería y Tecnología (16), Ciencias de la Salud (21), Educación y Humanidades (23), para sumar 191 instrumentos válidos.

Por otra parte, teniendo acceso a sujetos participantes en programas educativos de posgrado diferentes al universo de BUAP, se aplicó el instrumento a 31 sujetos sin adscripción a la BUAP, siempre y cuando cumplieran el requisito de ser PTC o estudiante de posgrado de sus respectivas IES. Estos sujetos fueron agrupados, independientemente del área de conocimiento a la que pertenecen, en una DES (estrato) que se ha llamado, para efectos de clasificación, "externa".

Como dato adicional, se menciona que estos últimos participantes son de adscripción a IES siguientes: Universidad Popular Autónoma del Estado de Puebla (UPAEP), Universidad Iberoamericana (IBERO) campus Puebla, Instituto de Estudios Universitarios (IEU) campus Puebla e Instituto Tecnológico de Estudios Superiores (ITESM) campus Puebla, principalmente.

Se aclara que el estudio se enfoca a las percepciones de sujetos de adscripción BUAP, por lo que el estudio se acota a esa Institución por la razón de ser referente a nivel nacional en México y, debido a ello, no se consideró una definición de muestra en sujetos externos a ella; sin embargo, la aplicación del instrumento a sujetos de IES externas como las mencionadas en párrafos previos se tomó más como un dato adicional de contraste, que pudiera considerarse eventualmente como un grupo de control.

Con los datos expuestos y, a manera de resumen, se encuentra que se aplicaron en total 267 instrumentos: 222 aplicados a individuos BUAP de 
los cuales 191 válidos y 45 no válidos; y 31aplicados a IES externas los cuales fueron válidos en su totalidad y sólo constituyen un referente que puede ser empleado como contraste.

\section{Instrumento}

Como parte de esta investigación, se diseña instrumento en modalidad de cuestionario, habiendo tomado como referencia central la teorías de competencias del docente de posgrado de Herrera (2017 y 2010) y Herrera et. al (2011) para aplicarse entre los sujetos de la muestra.

Datos de control del instrumento. Entre los datos de control cuestionario se incluye DES, estatus del sujeto (estudiante o PTC en funciones o con cargo administrativo), institución de adscripción, género, edad, grado académico, dominio de tecnologías de información y comunicación (TIC) y dominio de idiomas adicionales al materno.

Cuerpo del instrumento. El cuerpo del instrumento contiene 40 afirmaciones que responden a la pregunta inicial "Usted, ¿qué evaluaría a un docente de posgrado?, las cuales deben responderse asignando una de cinco opciones de la siguiente escala de Likert: 5) Muy importante para evaluar; 4) Importante para evaluar; 3) Más o menos importante para evaluar; 2) Poco importante para evaluar; y 1) Nada importante para evaluar. Posteriormente se solicita al participante que primeramente elija el "grado académico deseable" para docentes que participan en programas educativos de licenciatura; luego elija el "grado académico deseable" para docentes que laboran en programas educativos de especialidad; elija también el "grado académico deseable" para docentes que impartan en maestría; y, finalmente elija el "grado académico deseable" para aquel docente que participe en programas de doctorado. Las opciones de respuesta que se repiten para cada uno de los cuatro ítem mencionados son: Licenciatura, Especialidad, Maestría y Doctorado. También, como parte del cuerpo del instrumento, se plantean dos preguntas abiertas que se le pide responder: la primera sobre los indicadores que, a su juicio, son más relevantes y por tanto siempre deberían evaluarse en el docente de posgrado; la segunda pregunta abierta pregunta al encuestado sobre los 
indicadores que considera menos relevantes y, por tanto, no sería necesaria su evaluación en el docente que participa en programas de posgrado. El cuerpo del instrumento cierra con un espacio abierto para comentarios y sugerencias que el encuestado desee hacer, siempre que sean relativos a las características del docente de posgrado.

\section{Validez de la Prueba Piloto.}

Con apoyo del CIEP y autorización de la VIEP, se efectuó una prueba piloto, con la aplicación aleatoria del instrumento a 20 individuos quienes reunían el perfil de participante, para evaluar su efectividad, entendimiento, posibles confusiones y confirmar que las preguntas fueran adecuadas. Derivado de este primer pilotaje se realizaron adecuaciones al instrumento, principalmente en el tipo de lenguaje usado, ya que, debido a los tecnicismos, algunos participantes, principalmente estudiantes de maestría, no comprendían en su totalidad algunos ítem. Posteriormente a las adecuaciones mencionadas, se volvió a pilotear el instrumento con 10 personas más, encontrándose que se comprendía cabalmente ya había una definición precisa de cada ítem (indicadores de competencia) y, por tanto, se encontraba listo para aplicarse a la muestra.

\section{Validez de Expertos.}

El instrumento diseñado por la autora de este trabajo fue sometido a la retroalimentación de los miembros del Consejo de Investigación y Estudios de Posgrado (BUAP, 2010c), perteneciente a la Vicerrectoría de Investigación y Estudios de Posgrado (VIEP) de la BUAP. Los consejeros de Investigación y Estudios de Posgrados deben estar contratados como Profesor-Investigador Titular, tener una antigüedad mínima de cinco años en la Universidad como miembro del personal académico y haber desarrollado labores de investigación o docencia de posgrado en forma regular en los últimos tres años, como mínimo. El CIEP está integrado por docentes de posgrado de diversas DES y Facultades de la BUAP, quienes cuentan con diversidad de perfiles de formación (áreas del conocimiento de física, matemáticas, biología, química, administración, comunicación, artes, derecho y economía, por citar algunos); la mayoría son miembros del 
Sistema Nacional de Investigadores y participan en programas de posgrado pertenecientes al Programa Nacional de Posgrado de Calidad (PNPC) de CONACYT.

La participación de expertos con esos perfiles, quienes retroalimentaron a lo largo del periodo de estudio, el fondo y la forma del instrumento, además de garantizar su balance, cobertura y multidisciplinariedad, otorgaron características de pertinencia y validez al modelo de competencias e indicadores propuesto por la autora.

\section{Resultados}

Después de aplicar los análisis estadísticos mencionados someramente en el último párrafo de la Metodología, es conveniente presentar resultados y discutir sobre los hallazgos más relevantes de esta investigación.

\section{Análisis Descriptivo de los Encuestados.}

De 222 instrumentos aplicados, el 86\% son adscritos BUAP y 14\% son externos a ella; $42.5 \%$ son mujeres y $57.5 \%$ son hombres; los rangos de edad: 20-30 años 36.2\%, 31-40 años 31.7\%, 41-50 años $17.1 \%$ y de 51 años en adelante $15.1 \%$; el $40.9 \%$ son Licenciados; el 39.1 Maestros; el 18.6 Doctores y 1.4\% Especialistas; DES: Ciencias Sociales y Económico Administrativas 24.8\%, Ciencias Exactas 23.9\%, Ciencias Naturales 10.3\%, Ingeniería y Tecnología 7.2\%, Ciencias de la Salud 9.5\%, Educación y Humanidades $10.3 \%$ y Externa $14 \%$; estudiantes de maestría $42.5 \%$, de doctorado el $25.1 \%$, docentes de programas de posgrado $16.4 \%$ y personal que ocupa puestos directivos o administrativos en programas de posgrado $16.0 \%$; idioma castellano como materno 99\%, dominio del idioma inglés (superior al 75\%) $92.9 \%$; y en un $64.2 \%$ en promedio, usan programas y equipo de cómputo, bases de datos, plataformas educativas y TIC en general son dominio superior al $75 \%$. Los participantes requieren que el docente que imparte en licenciatura y especialidad posea grado de maestro, y grado de doctor para quien imparte en maestría y doctorado. 


\section{Análisis de Confiabilidad del Instrumento y Grupos de Indicadores de Competencias}

Al aplicar la prueba de Alfa de Cronbach (Welch y Comer, 1988, consultados en Universitat de València, 2013) a los 222 instrumentos capturados, se obtiene un coeficiente de confiabilidad o estabilidad de la escala de Likert de sus 40 ítem que componen cada instrumento en el rango de "excelente" con puntaje de 0.931 , arrojando 219 casos válidos y sólo 3 casos excluidos. Es conveniente aclarar que al aplicar esta prueba, el propio SPSS discriminó a tres de los instrumentos, mismos que señala como excluidos, por encontrar información no congruente o alineada a los fines de la investigación, sin embargo, por ser un número poco representativo de la muestra, no se ven afectados los resultados y el instrumento se mantiene dentro del criterio de excelente confiabilidad.

\section{Análisis de Confiabilidad por Grupos Ítem (indicadores de competencias)}

Al análisis de confiabilidad o estabilidad, también conocido como análisis de valoración cualitativa de los ítem (indicadores de competencias específicas) que componen el instrumento, se encontró que los ítem/indicadores de competencias específicas, que se asociaron por el propio SPSS a las competencias en sentido global o genérico son: aceptable para competencias I, V, VI y VII; bueno para competencias IX y X; suficiente para competencias II, III y VIII; e inaceptable para la competencia IV.

En cuanto a la valoración arrojada por el análisis de confiabilidad para cada ítem (indicadores de competencia específica) se encontró que de los 40 ítem: 2 se marcaron como excelentes, 20 muy buenos, 15 buenos, 2 regulares (participación como supervisor de prácticas profesionales y como coordinador de tutores) y sólo uno como insuficiente (participación como consejero universitario). Estos datos confirman que los sujetos de la muestra otorgan pertinencia a más del $92 \%$ de los indicadores de competencias del modelo sujeto de estudio. Estas últimas valoraciones se asocian a la representatividad de sujetos que son estudiantes de maestría y doctorado (67.6\%), para quienes los indicadores de competencias 
mencionadas no tan significativas como lo son para el propio docente o para PTC que se desempeñan en puestos administrativos o directivos $(32.4 \%)$, por representar indicadores de competencias específicas de gestión institucional.

\section{Análisis Comparativo}

El análisis de comparación, por su parte, se realizará empleando análisis comparativo de dos muestras y análisis comparativo para más de dos muestras.

\section{Análisis Comparativo para dos Muestras}

Al aplicar la Prueba T para muestras independientes, se consideró pertinente comparar las respuestas de los participantes por género $\mathrm{y}$ pertenencia institucional. Al comparativo de género se encontraron resultados no significativos para la mayoría de los 40 ítem, excepto para Facilidad de expresión verbal (C-IV) y Formar al estudiante involucrando valores (C-VI), los cuales son significativamente más importantes en el perfil del docente de posgrado, desde la perspectiva de las mujeres, respecto de los hombres; mientras que el ítem Generación y aplicación del conocimiento $(\mathrm{C}-\mathrm{X})$ es más significativo para los hombres, como expectativa en el perfil docente. Al comparativo de adscripción se encontró que para los adscritos a la BUAP, los indicadores de Participación del Comité Tutorial $(\mathrm{C}-\mathrm{V})$ y Gestión de proyectos de investigación $(\mathrm{C}-\mathrm{X})$ son significativamente más importantes en el perfil del docente de posgrado, que para aquellos que pertenecen a otras IES; mientras que los encuestados adscritos a alguna IES diferente a la BUAP, consideran significativamente más importantes en el perfil del docente de posgrado los ítem (indicadores de competencias) como Manejo de bases de datos y plataformas educativas (C-VII), Participación como Consejero Universitario (C-IX) y Asistencia a cursos de actualización disciplinaria o formación docente $(\mathrm{C}-\mathrm{X})$.

\section{Análisis Comparativo de Más de Dos Muestras (ANOVA)}


Se realizó el análisis comparativo ANOVA, bajo el método Scheffé, para estudiar a un primer grupo en función de su grado académico y a un segundo grupo en función de su DES de adscripción.

En el primer grupo se encontró que de los 40 ítem ligados a indicadores, en 11 de ellos los encuestados tuvieron respuestas con diferencias significativas, en cuanto a las percepciones de estos sujetos relativas a la importancia de igual número de indicadores de competencia que conforman el cuestionario:

- Para los sujetos con licenciatura es más significativa la competencia de Facilidad de expresión verbal de la C-IV.

- Para los sujetos con licenciatura, seguidos por los sujetos con maestría, son más significativas las competencias siguientes: Disposición para resolver dudas (C-I); Retroalimentación a las evaluaciones (C-III); Actividades de vinculación con los sectores productivos y sociales y Supervisión de práctica profesional (ambos de la C-VIII); Asistencia a cursos de actualización disciplinaria o formación docente $(\mathrm{C}-\mathrm{X})$; Participación como coordinador de tutores por área de conocimiento y Coordinación de posgrado (ambos de la C-IX).

- Para los sujetos con maestría, seguidos por los sujetos con licenciatura es más significativo el indicador de competencia de Estudio de la materia a través de casos y prácticas (intersección de C-I y C-II).

- Para los sujetos con doctorado, seguidos por los sujetos con maestría son más significativos los indicadores de competencias siguientes: Generación y aplicación del conocimiento $(\mathrm{C}-\mathrm{X})$; Participación del docente de posgrado como integrante de comité tutorial $(\mathrm{C}-\mathrm{V})$.

En el segundo grupo se encontró que de los 40 ítem ligados a indicadores, en 12 de ellos, los encuestados tuvieron respuestas que presentan diferencias significativas en sus percepciones sobre la importancia de igual número de indicadores de competencias que conforman el cuestionario.

Un patrón relevante encontrado con este análisis es que para los encuestados de la DES de Ciencias de la Salud es más significativa la posesión de ciertas competencias en el perfil del docente de posgrado respecto del resto de las DES, pues de los doce ítem que presentan valores 
significativamente diferentes, once corresponden a los otorgados como muy importantes por los encuestados del área de la salud. Conociendo el dato anterior, es más conveniente que se expongan a continuación los resultados de significancia menores otorgados por DES.

- Ciencias Exactas: Estudio a través de casos y prácticas y Materiales didácticos preparados para sus estudiantes (ambas de C-I y C-II); forma al estudiante involucrando valores y Es incluyente de grupos e ideologías y culturas diversas (ambas de C-VI); manejo de base de datos y plataformas educativas (C-VII); Asistencia a cursos de actualización disciplinaria o formación docente $(\mathrm{C}-\mathrm{X})$.

- Ingeniería y Tecnología: Participación como coordinador de tutores, Dirección de alumnos en proyectos de investigación y Participación en proyectos institucionales (todas de C-IX).

- Educación y Humanidades: Estudio a través de casos y prácticas (C-I y C-II, con la misma significancia que la DES de Ciencias Exactas); Actividades de vinculación con los sectores (C-VIII).

- Ciencias Naturales: Facilidad de expresión verbal (C-IV).

- Externos a BUAP: Participación de Comité Tutorial (C-V).

\section{Análisis factorial}

Una prueba estadística final que se aplica a la muestra de este trabajo es el análisis factorial, el cual es pertinente para contar con un análisis de integración o confirmatorio que, sumando a los hallazgos expuestos, le otorgue validez al modelo propuesto. Esta prueba que se compone por el contraste en el modelo factorial y la rotación de los componentes, se basa en la reducción de dimensiones de la muestra para cuantificar la comunalidad que comparte una variable (indicador de competencia) con el resto de ellas.

Es muy relevante la interpretación que se obtiene de la aplicación de análisis factorial a la muestra, bajo las técnicas de contraste en el modelo (adecuación muestral), extracción de componentes (comunalidades) y rotación de los componentes (correlaciones).

Con la primera y segunda técnicas se encontró que los 40 ítem guardan entre sí una relación notable y que las comunalidades entre ellos se 
encuentran en un rango de 0.818 (significativa) y un mínimo de 0.479 , siendo significativa para la mayoría de los ítem.

Con la tercera técnica se recurrió a la rotación de los componentes (ejes) bajo el método VARIMAX en diez grupos (número asignado automáticamente por SPSS), bajo los cuales se analizaron los 40 ítem/indicadores (variables) del instrumento. Los hallazgos de esta última técnica giran en torno a las altas correlaciones existentes entre los 40 indicadores de competencia del modelo propuesto (Herrera, 2017) y el acomodo en diez grupos definidos automáticamente por el propio método. Este último dato es sumamente relevante, puesto que la forma en que quedaron agrupados los 40 ítem/indicadores de competencias derivado de este análisis es muy similar al que se propone en el modelo de diez competencias para el docente de posgrado, y, aunque presenta muy ligeras variaciones en cuanto a agrupación de ítem, especialmente para la C-IV, estas se atribuyen más a la interpretación de ciertos indicadores en el perfil del docente de posgrado por los sujetos participantes, principalmente las relacionadas a IES de adscripción y estatus (estudiante o PTC).

Los datos numéricos que muestran la correlación y agrupaciones de indicadores de competencia pueden consultarse a detalle en la Tabla 1 denominada, "Análisis factorial: matriz de componentes rotados de indicadores de competencias", de elaboración propia de la autora (acorde a parámetros de REMIE en archivo adjunto).

Este cuadro muestra los resultados del análisis de componentes principales (ACP) a través de la rotación de los componentes (ejes) bajo el método VARIMAX para encontrar la correlación entre indicadores de competencia (40 variables) agrupados en diez grupos definidos automáticamente por el propio método. La primera columna numera a los 40 ítem /indicadores de competencia; la segunda columna indica el número de competencia a la cual pertenece cada indicador en el modelo de Herrera (2017); en la tercera columna se muestra la proporción de correspondencia entre los indicadores de competencia según el modelo referido, respecto del acomodo automático del análisis factorial bajo el método de componentes rotados; la cuarta columna enlista los indicadores de competencia obtenidos referidos en la tercera columna; y, las columnas cinco a la catorce muestran las correlaciones entre los indicadores de competencia analizados y su acomodo por grupo de pertenencia, encontrados por el análisis estadístico. 


\section{Herrera-Decálogo de Competencias Universidad}

Tabla 1:

"Análisis factorial: matriz de componentes rotados de indicadores de competencias"

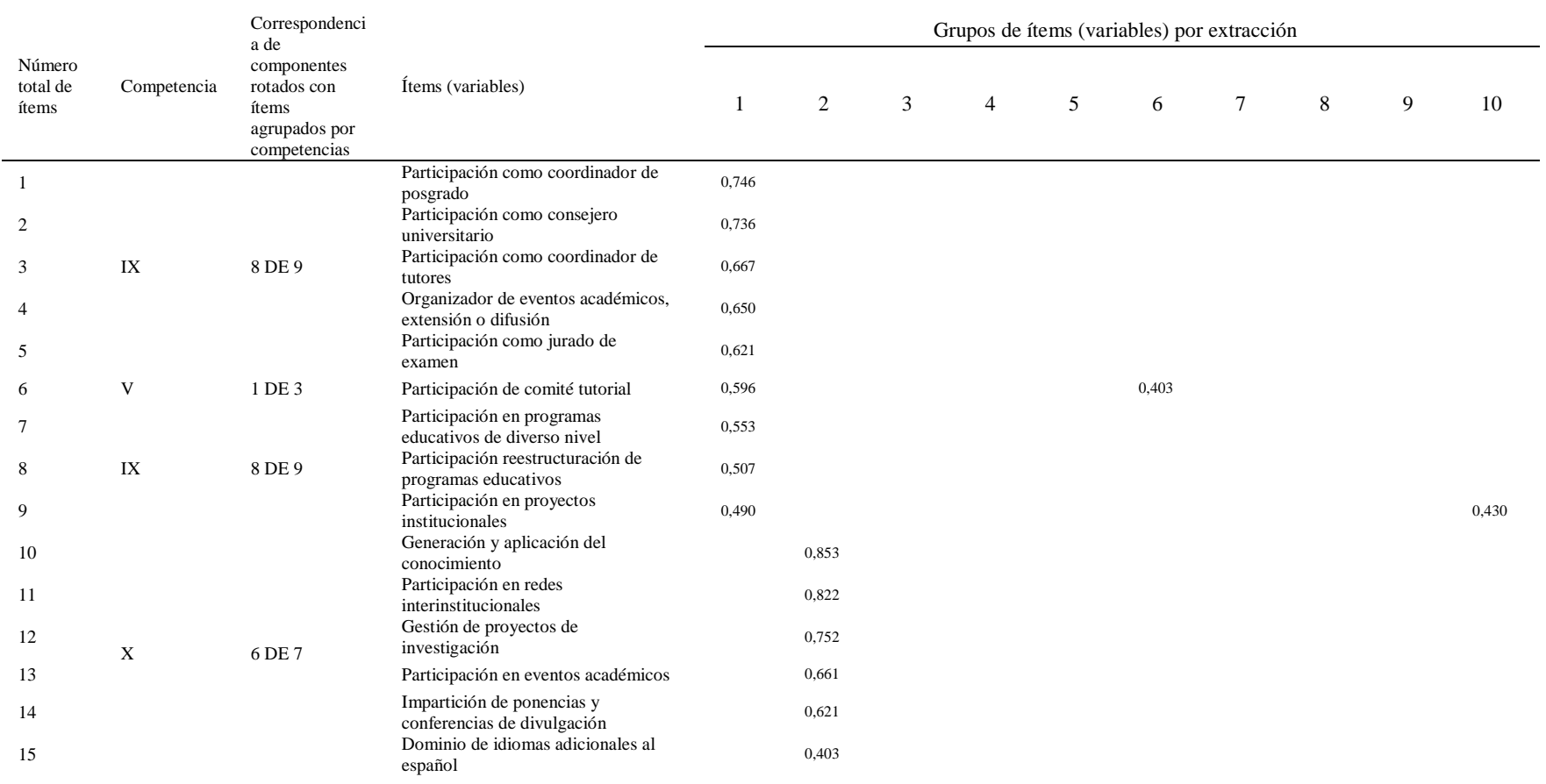


Tabla 1:

"Análisis factorial: matriz de componentes rotados de indicadores de competencias"

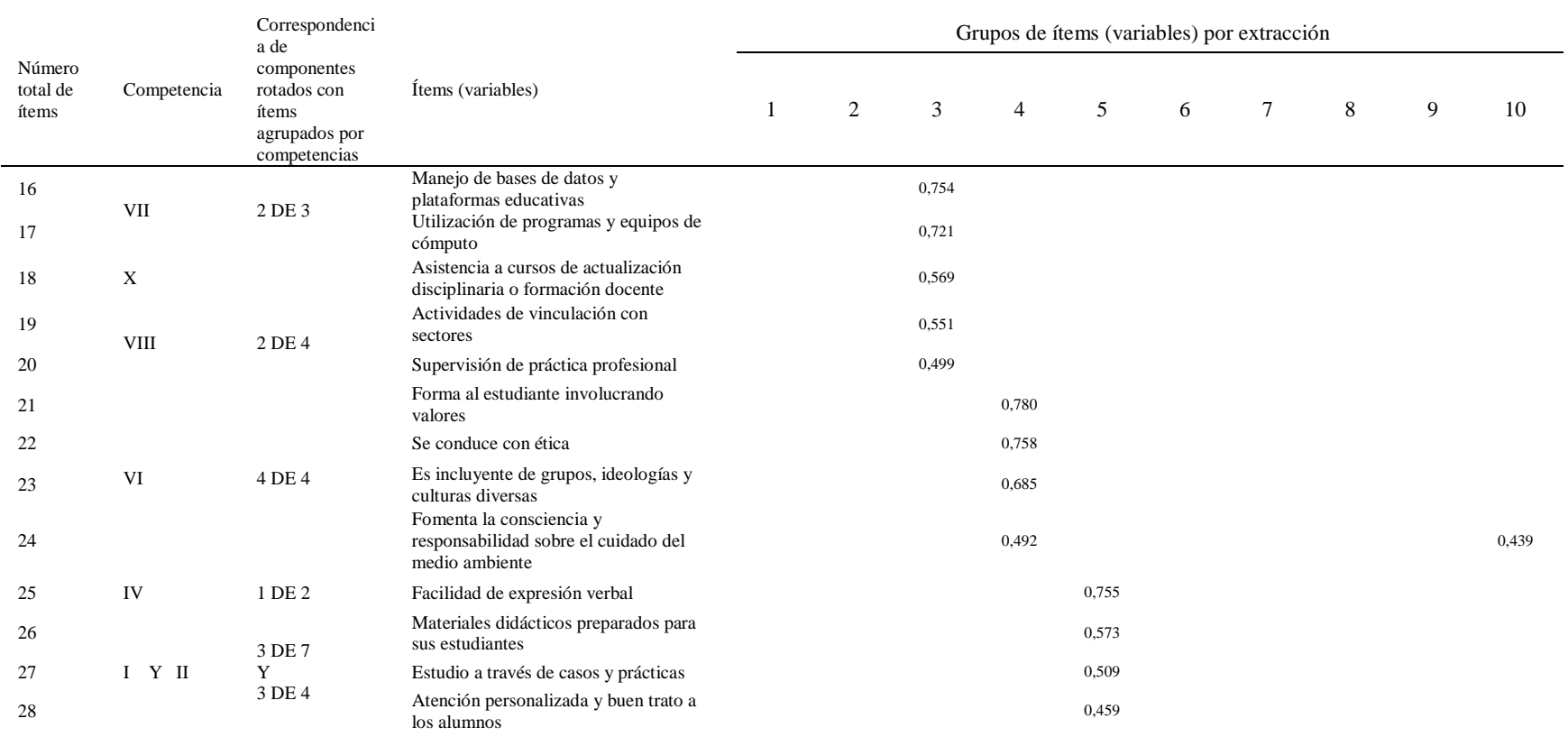




\section{Herrera-Decálogo de Competencias Universidad}

Tabla 1:

"Análisis factorial: matriz de componentes rotados de indicadores de competencias"

\begin{tabular}{|c|c|c|c|c|c|c|c|c|c|c|c|c|c|}
\hline \multirow{2}{*}{$\begin{array}{l}\text { Número } \\
\text { total de } \\
\text { ítems }\end{array}$} & \multirow{2}{*}{ Competencia } & \multirow{2}{*}{$\begin{array}{l}\text { Correspondenci } \\
\text { a de } \\
\text { componentes } \\
\text { rotados con } \\
\text { ítems } \\
\text { agrupados por } \\
\text { competencias } \\
\end{array}$} & \multirow{2}{*}{ Ítems (variables) } & \multicolumn{10}{|c|}{ Grupos de ítems (variables) por extracción } \\
\hline & & & & 1 & 2 & 3 & 4 & 5 & 6 & 7 & 8 & 9 & 10 \\
\hline 29 & viii & 2 de 4 & $\begin{array}{l}\text { motivación al alumno para aprender } \\
\text { por sí mismo }\end{array}$ & & & & & & 0,718 & & & & \\
\hline 30 & iv & 1 de 2 & $\begin{array}{l}\text { disposición a escuchar exposiciones, } \\
\text { criticar y guiar }\end{array}$ & & & & & & 0,560 & & & & \\
\hline 31 & viii & 2 de 4 & fomento del trabajo en equipo & & & & & & 0,518 & & & & \\
\hline 32 & \multirow{2}{*}{ iii } & \multirow{2}{*}{2 de 2} & da a conocer criterios de evaluación & & & & & & & 0,828 & & & \\
\hline 33 & & & retroalimentación a las evaluaciones & & & & & & & 0,653 & & & \\
\hline 34 & \multirow{3}{*}{$\mathrm{i}$} & \multirow{3}{*}{3 de 7} & $\begin{array}{l}\text { dominio y preparación de contenidos } \\
\text { de materia }\end{array}$ & & & & & & & & 0,777 & & \\
\hline 35 & & & asistencia y puntualidad & & & & & & & & 0,713 & & \\
\hline 36 & & & disposición para resolver dudas & & & & & & & & 0,506 & & \\
\hline 37 & \multirow{2}{*}{$\mathrm{v}$} & \multirow{2}{*}{2 de 3} & participación como asesor & & & & & & & & & 0,834 & \\
\hline 38 & & & participación como tutor & & & & & & & & & 0,758 & \\
\hline 39 & ix & 1 de 9 & $\begin{array}{l}\text { dirección de alumnos en proyectos de } \\
\text { investigación }\end{array}$ & & & & & & & & & & 0,678 \\
\hline 40 & $\mathrm{i}$ & 1 de 7 & $\begin{array}{l}\text { enriquecimiento de la clase con } \\
\text { comentarios y ejemplos }\end{array}$ & & & & & & & & & & 0,492 \\
\hline
\end{tabular}




\section{Conclusiones}

\section{Modelo de perfil del docente de posgrado: diez competencias y sus indicadores}

Lo hallazgos expuestos y discutidos en el apartado de resultados confirman la validez, confiabilidad y pertinencia al modelo de gestión de capital humano universitario: indicadores de competencias en el perfil del docente de posgrado.

La exposición de resultados y su discusión son prueba de que existe congruencia entre competencias e indicadores de competencia que, tanto agrupados, como en su conjunto, conforman un decálogo de competencias innovador para el docente que imparte en posgrado, el cual se enlista a continuación, mencionando cada una de las diez competencias, seguidas de los indicadores de competencia asociados a cada una de ellas.

- Competencia I (C-I). Gestionar dinámicamente la metodología del proceso de enseñanza-aprendizaje: asistencia y puntualidad; dominio y preparación de contenidos de materia; y, disposición para resolver dudas.

- Competencia II (C-II). Realizar planes de materia considerando la diferenciación/inclusión de los alumnos: atención personalizada y buen trato a los alumnos; enriquezca la clase con comentarios y ejemplos; estudio a través de casos y prácticas; y, preparación de materiales didácticos para sus estudiantes.

- Competencia III (C-III). Elaborar dispositivos de evaluación acordes a objetivos planeados y expectativas de aprendizaje de sus estudiantes: dé a conocer los criterios de evaluación; y, retroalimentación a las evaluaciones.

- Competencia IV (C-IV). Comunicación eficiente y eficaz, respetando derechos y valores: facilidad de expresión verbal; y, disposición a escuchar las exposiciones de sus estudiantes, criticarlos y guiarlos.

- Competencia V (C-V). Ofrecer tutorías y acompañamiento a los estudiantes: participación como tutor (guía en el medio universitario); participación como asesor (guía en temas académicos que pueden incluir el acompañamiento en la elaboración de trabajos de investigación para titulación); y, participación en comité tutorial. 
- Competencia VI (C-VI). Fomentar en la formación de los alumnos principios éticos, valores, inclusión y ecología: que el docente se conduzca con ética; forme al estudiante involucrando valores; incluyente de grupos, ideologías y culturas diversas; y, fomentar la consciencia y responsabilidad sobre el cuidado del medio ambiente.

- Competencia VII (C-VII). De utilizar las nuevas tecnologías de la información y comunicación: preparación y uso de materiales didáctico visual y audiovisual para sus estudiantes (con el mismo ítem del cuestionario que ya se analizó en la Competencia número II); utilización de programas y equipos de cómputo; y, manejo de bases de datos y plataformas educativas.

- Competencia VIII (C-VIII). Fomentar situaciones de auto aprendizaje, vinculación y trabajo en equipo: fomento de trabajo en equipo; motivación del docente hacia sus alumnos para aprender por sí mismos; actividades de vinculación con los sectores productivos y sociales; y, supervisión de prácticas profesionales.

- Competencia IX (C-IX). Participar en la gestión de la institución: participación en la reestructuración de programas educativos; participación en programas educativo de diverso nivel; participación como coordinador de tutores; participación como jurado de examen; dirección de alumnos en proyectos de investigación; participación en proyectos institucionales; participación como coordinador de posgrado; participación como consejero universitario; y, organización de eventos académicos, de extensión o difusión.

- Competencia X (C-X). Autoevaluación permanente y desarrollo continuos: grado académico idóneo para los docentes de Maestro para impartir en licenciatura y especialidad y de Doctor para impartir en maestría y doctorado; dominio de idiomas adicionales al español; asistir a cursos de actualización disciplinaria o formación docente; participación del docente de posgrado con ponencias y conferencias en eventos con fines de divulgación; participación en eventos académicos; generación y aplicación del conocimiento (entendida como la producción científica, derivada de actividades de investigación -libros, capítulos de libro, artículos, memorias, desarrollos tecnológicos, patentes-); participen en redes interinstitucionales; y, gestión de proyectos de investigación. 
El alcance de este modelo se extiende más allá de sus efectos de evaluación al desempeño en una IES específica, ya que en la medida que sus competencias e indicadores están validados para formar parte del perfil del docente que participa en programas educativos de posgrado, son parámetro para realizar el resto de las funciones propias de la $\mathrm{ACH}$ para este actor, en diversas IES, con el sustento del rigor científico que le otorga la presente.

\section{Referencias}

Arias G. y Heredia E. (1999). Administración de Recursos Humanos para el alto desempeño, Trillas. México.

Bain, Ken. (2007). Lo que hacen los mejores profesores universitarios. Traducción de Oscar Barberá. 2a . Edición. Publicacions Universitat de València.

BUAP. (2002). Programa Institucional de Evaluación Académica (PIEVA). Retrieved from http://cmas.siu.buap.mx/portal_pprd/wb/pieva/vision

BUAP. (2006). Las políticas y tendencias dominantes en el contexto nacional e internacional que influyen en la educación superior. Fundamentos del Modelo Universitario Minerva (MUM), pp. 12-17. México. Retrieved from http://transversalidadmum.wikispaces.com/file/view/0I_Fundamentos.pdf

BUAP. (2008). Convocatoria al Programa de Estímulos al Desempeño del Personal Docente. Retrieved from http://acreditacion.cs.buap.mx/acreditacion_ICC/Documentos/Informacio n\%20Profesores\%20FCC/convocatoria\%20ESDEPED.pdf

BUAP. (2009). Matrícula de posgrado BUAP: de las más grandes del país. Retrieved from http://www.poblanerias.com/2009/07/matricula-deposgrado-buap-de-las-mas-grandes-del-pais/

BUAP. (2010a). Anuario Estadístico Institucional 2010. Dirección General de Estudios de Posgrado. Retrieved from https://www.yumpu.com/es/document/view/17383420/anuarioestadistico-institucional-2010-2011-benemerita- 
BUAP. (2010b). Foro de Seguimiento y Consolidación del Posgrado. Vicerrectoría de Investigación y Estudios de Posgrado (VIEP). Metepec, Puebla. Marzo 2010.

BUAP. (2010c). Integración del Consejo de Investigación y Estudios de Posgrado (CIEP). Retrieved from http://www.viep.buap.mx/ciep/ciepintegracion.php

Bunk, G. P. (1994). La transmisión de las competencias en la formación y perfeccionamiento de profesionales. Revista Europea de Formación Profesional. En CEDEFOP. No 1.

Cano, E. (2005). Cómo mejorar las competencias de los docentes. Editorial Graó. Barcelona, España.

Comellas, M. (2002). Las Competencias del Profesorado para la acción tutorial. Editorial Wolters Kluwer Education. ISBN 9788471977366.

CONACYT (2012). Programa Nacional de Posgrados de Calidad.

Retrieved from http://conacyt.gob.mx/index.php/becas-yposgrados/programa-nacional-de-posgrados-de-calidad

Cuesta, A. (2005). Tecnología de gestión de recursos humanos. $2^{\mathrm{a}}$. Edición. Editorial Academia. La Habana, Cuba.

Echeverría, B. (2002). Gestión de la competencia de acción profesional. Revista de Investigación Educativa, 20 (1), 7-43. Retrieved from http://revistas.um.es/rie/article/view/97411/93521

EEES. (1999). Declaraciones de Bolonia. Retrieved from http://www.educacion.gob.es/boloniaensecundaria/img/Declaracion_Bolo nia.pdf

EEES. (2009). Declaración de Leuven and Louvain-la-Neuve. Retrieved from

http://www.une.es/media/Ou1/File/DocumentosEEES/lovaina_abril_09.p df

Gonczy, A. (2001). Análisis de las tendencias internacionales y de los avances en educación y capacitación laboral basadas en normas de competencias. En A. Argüelles y A. Gonczy, Educación y capacitación basadas en normas de competencias (pp. 38-40). México: Limusa.

Herrera, A. (2010). Propuesta de un instrumento de evaluación docente por alumnos de posgrado, con enfoque de competencias. Foro de Seguimiento y Consolidación del Posgrado. Vicerrectoría de Investigación y Estudios de Posgrado (VIEP), BUAP. Metepec, Puebla. 
Herrera, A., González, M. y Djordjevic, D. (2010a). Estandarización Internacional y Normativa del Perfil Docente en Instituciones de Educación Superior en México. Memorias del II Congreso Internacional de Administración de Empresas 15-18 septiembre 2010. Escuela de Administración de Empresas de la Universidad Pedagógica y Tecnológica de Colombia (UPTC). ISSN: 2145-4795. Paipa-Boyacá. Colombia.

Herrera, A. y Djordjevic, S. (2010b). La Evaluación por Competencias del Docente de Posgrado. Memorias del VII Encuentro Participación de la Mujer en la Ciencia 26-28 mayo 2010. Clave S1HCC03. CIO. ISBN978607-95228-1-0. México.

Herrera, A., Djordjevic, D. y Djordjevic, S.V. (2011). Instrumento de Evaluación al Desempeño Docente de Posgrado bajo un Modelo por Competencias. Memorias del VIII Encuentro Participación de la Mujer en la Ciencia 18-20 mayo 2011. Clave S3HCC16. CIO. ISBN978-60795228-1-0. México.

Herrera, A. (2017). Un modelo de perfil para gestión de personal: diez competencias del docente de posgrado. Revista Global de Negocios (RGN), 5(8). Retrieved from http://bit.ly/2CoM2iA

Instituto Nacional de Empleo en España (INEM) (1995). Metodología para la ordenación de la formación profesional ocupacional. Subdirección general de gestión de formación ocupacional. Retrieved from www.uch.edu.ar/rrhh.

Lillo, A. et al. (2007). El capital humano como factor estratégico para la competitividad del sector turístico. Cuadernos de turismo, 19, 47-69. Retrieved from http://www.redalyc.org/articulo.oa?id=39801903

Lindgren, R., Henfridsson, O. y Schultze, U. (2004). Design principles for competence management research? Yes. Academy of Management Review, 26(1), 41-56. doi: 10.2307/25148646

López Mojarro, M. (1999). A la calidad por la evaluación. Madrid: Escuela Española.

Marín, H. (2002). Desarrollo de competencias en el docente universitario de acuerdo con las exigencias de los actuales procesos organizacionales.

Revista de Ciencias Sociales, 8(1), 183-194. Retrieved from http://produccioncientificaluz.org/index.php/rcs/article/view/13168/1315 3 
McClelland, D.C. (1973). Testing for competence rather than for intelligence. American Psycologist, 28(1), 1-14. Retrieved from https://www.therapiebreve.be/documents/mcclelland-1973.pdf

Medina, M. (2009). La formación profesional basada en competencias en la era del conocimiento y la información (Tesis de Doctor en Ciencias de la administración). Biblioteca de la Universidad Autónoma de México.

Medina, M., Armenteros, M., Guerrero, L. y Barquero, J. (2012). Las competencias gerenciales desde una visión estratégica de las organizaciones: un procedimiento para su identificación y evaluación del desempeño. Revista Internacional de Administración y Finanzas (RIAF), 5(2), 79-100. Retrieved from ftp://ftp.repec.org/opt/ReDIF/RePEc/ibf/riafin/riaf-v5n2-2012/RIAFV5N2-2012-6.pdf

Mobilia, H. y García-Valcárcel, A. (1997). Reflexiones sobre la calidad en la enseñanza superior. Revista Española de Pedagogía, 208, 509-524. Retrieved from http://www.jstor.org/stable/23764940?seq=1\#page_scan_tab_contents Morín, E. (1999). Los siete saberes necesarios para la educación del futuro. Editora Correo de la UNESCO, ISBN 9687474076.

Perrenoud, P. (2004). Diez nuevas competencias para enseñar. Invitación al viaje. Ed. Graó. España.

Rueda, M. (2008). La evaluación del desempeño docente en la universidad. Revista Electrónica de Investigación Educativa, 10. Retrieved from http://redie.uabc.mx/redie/article/view/196

Rueda, M. (2009). La evaluación del desempeño docente: consideraciones desde el enfoque por competencias. Revista Electrónica de Investigación Educativa, 11 (2). Retrieved from

http://redie.uabc.mx/redie/article/view/234

SEP (2013). Programa de Estímulos al Desempeño del Personal Docente. Retrieved from http://dsa.sep.gob.mx/informacionadicional.html SHCP (2002). Lineamientos Generales para la Operación del Programa de Estímulos al Desempeño del Personal Docente de Educación Media Superior y Superior. Secretaría de Hacienda y Crédito Público. Gobierno de México. México.

Tejada Fernández, J. (2005). El trabajo por competencias en el prácticum: cómo organizarlo y cómo evaluarlo. Revista electrónica de Investigación 
educativa, 7(2), 1-31. Retrieved from http://redie.uabc.mx/redie/article/view/192

Tejedor, F.J. y Jornet, J. M. (2008). La evaluación del profesorado universitario en España. Revista Electrónica de Investigación Educativa,10, 1-29. Retrieved from http://redie.uabc.mx/NumEsp1/contenido-tejedorjornet.html

Universitat de València, España. (2013). Alfa de Cronbach y consistencia interna de los ítems de un instrumento de medida. Retrieved from ttp://www.uv.es/ friasnav/AlfaCronbach.pdf

Uriel, E., Monfort, V., Ferri, J., y Fernández de Guevara, J. (2001). El sector turístico en España. Editorial Caja de ahorros del Mediterráneo. España. Zabalza, M. (2005). Competencias docentes del profesorado universitario. Calidad y desarrollo Profesional. Ed. Narcea. España.

Zabala, A. y Arnau, L. (2008). Evaluar competencias es evaluar procesos en la resolución de situaciones problema. En 11 Ideas clave: como aprender y enseñar competencias. Ed. Graó, $4^{\mathrm{a}}$ reimpresión 2008. Barcelona España. ISBN: 978-84-7827-500-7.

\begin{abstract}
Azucena Leticia Herrera es profesora de la División de Negocios de la Universidad Tecnológica de Puebla de México.

Contact Address: División de Negocions en Antiguo Camino a La Resurrección 1002 - A, Zona Industrial, 72300 Puebla, Pue., México
\end{abstract}

E-mail: azulether@hotmail.com 